\title{
Triagem de Indicadores de Altas Habilidades/Superdotação: Estrutura Fatorial
}

\author{
Tatiana de Cássia Nakano¹, Karina da Silva Oliveira \\ Pontifícia Universidade Católica de Campinas, Campinas-SP, Brasil
}

\section{RESUMO}

A relevância da avaliação das altas habilidades/superdotação (AH/S) pode ser compreendida dentro de uma proposta que busca a valorização dos pontos fortes, aptidões e talentos dos indivíduos, assim como a prevenção de problemas sociais e emocionais decorrentes da ausência de identificação. Nesse sentido, pode-se afirmar sua proximidade com os pressupostos da Psicologia Positiva. Perante a lacuna de instrumentos específicos para avaliação desse fenômeno, a construção de uma escala de triagem de indicadores de AH/S foi iniciada. Dentre os estudos para investigação de suas qualidades psicométricas, o presente estudo apresenta os resultados da análise fatorial confirmatória. As respostas de 216 avaliações, feitas pelos professores de estudantes do $2^{\circ}$ ao $9^{\circ}$ ano do ensino fundamental, foram analisadas. Índices de ajuste adequados foram encontrados, confirmando um modelo teórico multidimensional, que abrange, na escala, cinco domínios em que a AH/S pode se manifestar: capacidade intelectual geral, habilidades acadêmicas específicas, liderança, criatividade e talento artístico.

Palavras-chave: validade do teste; testes psicológicos; superdotado.

\section{ABSTRACT - Giftedness Indicators Screening Scale: factorial structure}

The relevance of the assessment of giftedness can be understood within a proposal that seeks to highlight the strengths, aptitudes and talents of individuals, aiming for the prevention of social and emotional problems resulting from lack of identification. Accordingly, its proximity to the ideas of Positive Psychology can be affirmed. Considering the lack of specific instruments for evaluating this phenomenon, the development of a screening scale of indicators of giftedness was initiated. Among the studies to investigate its psychometric qualities, the present study presents the results of the confirmatory factorial analysis. The data from 216 evaluations, performed by teachers of students of grades 2 to 9 of elementary education, were analyzed. Adequate fit indices were found, confirming a multidimensional theoretical model, composed of five domains in which giftedness can be manifested: general intellectual ability, specific academic abilities, leadership, creativity and artistic talent.

Keywords: test validity; psychological tests; gifted children.

\section{RESUMEN - Escala de Identificación de las Altas Capacidades/Superdotación: Estructura factorial}

La relevancia de la evaluación de las altas capacidades/superdotación (AH/S) puede ser comprendida dentro de una propuesta que busca la valorización de los aspectos positivos, aptitudes y talentos de los individuos, así como la prevención de problemas sociales y emocionales derivados de la ausencia de identificación. En ese sentido, se puede afirmar su cercanía con las suposiciones de la Psicología Positiva. Ante la falta de instrumentos para la evaluación de este fenómeno, se inició la construcción de una escala de identificación de altas capacidades. Entre los estudios para investigación de sus cualidades psicométricas, el presente objeto de investigación presenta los resultados del análisis factorial confirmatorio. Las respuestas de 216 evaluaciones, realizadas por los profesores de estudiantes del $2^{\circ}$ al $9^{\circ}$ año de la enseñanza primaria, fueron analizadas. Se han encontrado índices de ajuste adecuados, confirmando un modelo teórico multidimensional, que abarca cinco dominios en los que la superdotación puede manifestarse: capacidad intelectual general, habilidades académicas específicas, liderazgo, creatividad y talento artístico.

Palabras clave: validez de la prueba; pruebas psicológicas; superdotado.

Especificamente na Psicologia, as altas habilidades/superdotação (AH/S) têm se mostrado uma área cujo estudo precisa considerar o desenvolvimento de distintos construtos psicológicos que estão envolvidos em sua compreensão (Gallagher, 2008), bem como o fato de que os psicólogos vêm sendo, cada vez mais frequentemente, desafiados a fazerem predições sobre o potencial desses indivíduos. Estudos atuais, realizados em diferentes países, têm reconhecido as $\mathrm{AH} / \mathrm{S}$ como um fenômeno multidimensional, que envolve diversos elementos psicológicos, tais como inteligência, criatividade, liderança, motivação, psicomotricidade, habilidades artísticas e socioemocionais (Feldman, 2000; Gagné, 2005). Consequentemente, a performance superior 
pode ser identificada em uma série de áreas: acadêmica, artística, social, dentre outras (Costa \& Lubart, 2016), podendo ocorrer em uma única área ou em mais de uma (Brasil, 2010).

Essa concepção multidimensional tem guiado as políticas públicas nacionais e internacionais, de forma a reconhecer a existência de um elenco de características que se apresentam de forma permanente e notável no indivíduo, as quais proporcionam, a este, destaque em algum campo do conhecimento (Renzulli, 2004). Dada tal amplitude, os indivíduos com AH/S apresentam capacidade de se desenvolver em qualquer área potencialmente valiosa do desempenho humano (Guenther, 2006).

A avaliação das $\mathrm{AH} / \mathrm{S}$ deve ter, como objetivos principais, a identificação de indivíduos que apresentam algum tipo de habilidade acima da média (ou mais de um tipo). Independentemente das $\mathrm{AH} / \mathrm{S}$ ocorrerem de forma isolada ou em conjunto, Kerr e Sodano (2003) chamam a atenção para a necessidade de que a avaliação seja feita considerando-se o uso de instrumentos adequados, específicos para essa população. Tal necessidade se ampara na constatação de que não é incomum que esses indivíduos, por exemplo, alcancem o limiar máximo de pontuação que os instrumentos apresentam em suas tabelas normativas, visto que elas foram elaboradas para a população sem esse diagnóstico (Heller, 2004).

Para além do diagnóstico em si, a avaliação das $\mathrm{AH} / \mathrm{S}$ se mostra importante perante a possibilidade de que os resultados possam ser utilizados, principalmente, no planejamento de um atendimento diferenciado, desenvolvimento adequado de seu potencial e utilização dos dados para planejamento educacional e profissional (Sabatella, 2008). Tal processo não visa, diferentemente do que muitos imaginam, a "rotulação" desse indivíduo, nem tampouco a criação de um grupo privilegiado (Pocinho, 2009), mas sim o oferecimento de oportunidades adequadas de desenvolvimento e estimulação de suas habilidades, necessidades e interesses (Negrini \& Freitas, 2008).

Dadas as semelhanças, pode-se afirmar que a identificação das AH/S também visa, assim como a Psicologia Positiva, compreender os processos e fatores que podem promover um desenvolvimento psicológico sadio, dentro de uma visão mais positiva e que busca a adaptação, valorização das forças e qualidades positivas do indivíduo, assim como o fortalecimento dos recursos pessoais (Scorsolini-Comin \& Santos, 2010). No caso das AH/S, sua avaliação visa ainda à prevenção de dificuldades, principalmente sociais e emocionais que podem ser decorrentes da ausência de identificação, sendo tais aspectos, usualmente apontados como fonte de vulnerabilidades e dificuldades emocionais nessa população (Piske, 2017; Prado \& Fleith, 2017; Valadez, 2010). Com a identificação desses indivíduos, busca-se evitar o aumento dos fatores de risco (Chagas-Ferreira \& Sousa, 2018) tais como ansiedade, depressão, perfeccionismo negativo, problemas de conduta e dificuldades de relacionamento com os pares, usualmente relatados na literatura científica, dado o fato de que as necessidades, nessas áreas, se mostram mais intensas nessa população específica (Irueste, Saco, \& Nicolás, 2018).

As autoras afirmam que, nesse sentido, as avaliações e, posteriores intervenções, podem favorecer um desenvolvimento social, emocional, acadêmico e psicológico posterior mais saudável. Igualmente, prevenção, intervenção precoce, promoção da saúde, resiliência e invulnerabilidade vêm sendo destacadas como pontos importantes de serem trabalhados na temática (Pfeiffer, 2018). Segundo o autor, adolescentes superdotados, quando são envolvidos em trabalhos adequados com os propósitos citados anteriormente, usualmente relatam altos níveis de bem-estar, comportamentos sociais bem desenvolvidos, forças de caráter fortalecidas (usualmente empatia, compaixão, otimismo, senso de humor e amor pelo aprendizado), bem como facilidade em entender as emoções de outras pessoas e controlar suas próprias. Tais características, se fortalecidas, têm se mostrado responsáveis, ainda segundo o autor, por importantes diferenças na vida dos superdotados.

Assim, o conhecimento das áreas em que se encontram fortalecidas pode ser visualizado como um fator protetivo em relação a algum possível desajustamento, bem como auxiliar em processos relacionados à construção de um autoconceito positivo, no estabelecimento de valores, na melhoria da motivação, na autoestima e na estabilidade emocional (Chagas-Ferreira, 2014), construtos abarcados pela Psicologia Positiva.

A identificação das AH/S funcionaria, dentro dessa perspectiva, como um mecanismo de proteção na medida em que os resultados decorrentes permitiriam o planejamento de uma proposta de atendimento adequado, bem como o oferecimento de um ambiente propício ao desenvolvimento e fortalecimento dos aspectos positivos e potenciais que o indivíduo apresenta (Sabatella, 2008). Nesse sentido, a literatura tem demonstrado que, na presença de um ambiente adequado e de suporte, esses indivíduos geralmente não apresentam diferenças em relação à população geral no que se refere a questões emocionais e possíveis dificuldades derivadas (Freitas, Schelini, \& Pérez 2017).

Conduzida dessa maneira, a avaliação conseguiria mostrar-se eficaz na confirmação dos diagnósticos e na descrição do perfil psicológico do indivíduo (Almeida, Araújo, Sainz-Gómez, \& Prieto, 2016), fomentar o desenvolvimento das potencialidades, fornecer suporte às fragilidades relacionadas ao desenvolvimento e ao comportamento, bem como auxiliar na decisão acerca das medidas educativas mais adequadas ao sujeito (Almeida, Fleith, \& Oliveira, 2013). É com base nessa abordagem que o estudo aqui pode ser descrito dentro do enfoque da Psicologia Positiva, se considerar que a identificação deve ser vista como um processo contínuo, um conjunto de 
habilidades que emergem e se desenvolvem à medida em que a criança amadurece e que deve, preferencialmente, apontar os pontos fortes, aptidões e talentos de cada criança, em detrimento de suas fraquezas e incapacidades (Virgolim, 1997).

Nesse sentido, reforça-se a necessidade de que o processo de identificação privilegie uma visão sistêmica e global do indivíduo avaliado (Guimarães, 2007). No entanto, na prática, tal situação não tem sido encontrada. Embora diversas leis federais venham propondo, há vários anos, a importância de oferecimento de ações de intervenção voltadas ao atendimento de crianças com altas habilidades, a dificuldade em determinar quem seriam essas crianças e, principalmente, de criar formas para avaliá-las apropriadamente, tem atuado de forma a impedir que muitas delas tenham acesso a programas de estimulação e desenvolvimento, devendo ser esse um dos desafios atuais da área da avaliação psicológica.

Buscando responder às dificuldades inerentes à avaliação de um fenômeno tão complexo, esforços para identificar alunos superdotados e talentosos têm sido ampliados, tanto aqueles que visam à adequação de instrumentos já existentes, como outros com o objetivo de complementar o processo avaliativo por meio da inserção de outros olhares possíveis (avaliações externas feitas por pais e professores, avaliação de produções). Isso porque, no Brasil, há carência de medidas válidas para identificar alunos com AH/S (Barbosa, Schelini, \& Almeida, 2012), quando comparado a outros países. Faltam, principalmente, instrumentos elaborados especificamente para a identificação das $\mathrm{AH} / \mathrm{S}$ dentro de uma compreensão multidimensional do fenômeno, visto que os testes comumente utilizados foram construídos para uso na população geral, e podem não se mostrarem eficazes em capturar altos níveis de habilidades presentes nesses indivíduos, muito menos suas singularidades (Almeida et al., 2016), bem como ausência de medidas relacionadas a uma série de domínios, tais como o psicomotor e o socioemocional (Freitas et al., 2017).

Perante esse quadro e a constatação de que a ausência de identificação pode gerar uma série de dificuldades ao indivíduo, dentre as quais, problemas de ajustamento, falta de interesse, baixo desempenho ou insucesso escolar e problemas socioemocionais (Martins et al., 2016), as quais podem colocá-lo em situação de risco acadêmico, social e emocional, o processo de construção de uma escala de Triagem de Indicadores de Altas Habilidades/Superdotação (TIAH/S) foi iniciado, cujo uso foi pensado como uma ferramenta para ser usada por professores e demais profissionais que atuam junto a essa população, de modo a se constituir em um recurso auxiliar para a indicação dos indivíduos para um processo amplo de avaliação.

Nesse sentido, a ferramenta se constitui como um instrumento de screening (sondagem) a ser utilizado em uma fase inicial de despiste, constituindo-se em um instrumento de aplicação rápida e coletiva, sendo que, a partir de seus resultados, os professores e demais profissionais poderão nomear aqueles alunos que possivelmente apresentem sinais indicativos da presença de um quadro de $\mathrm{AH} / \mathrm{S}$, os quais deverão ser encaminhados para uma avaliação psicológica mais ampla e aprofundada. Assim, o instrumento não deve ser utilizado como instrumento de avaliação, diagnóstico ou orientação psicopedagógica, de modo que, em hipótese nenhuma, seu resultado substitui um processo de identificação conduzido por um psicólogo, por meio do uso de métodos e técnicas de uso restrito a esse profissional.

Nessa fase de nomeação, um dos instrumentos mais utilizados internacionalmente são escalas respondidas por professores, baseadas na hipótese de que esses estudantes possuem diferentes características comportamentais de seus pares em relação aos seus aspectos cognitivos, físicos, socioemocionais e pessoais (Sahin, 2013). Este tipo de instrumento tem sido apontado como o mais usado para o rastreio de superdotação nos Estados Unidos (Renzulli, Siegle, Reis, Gavin, \& Reed, 2009), dado seu potencial enquanto ferramenta de observação sistematizada de uma grande variedade de habilidades dos estudantes (Cleveland, 2017; Kaufman, Plucker, \& Russell, 2012), aumentando a amplitude do processo de identificação (Gray, 2007).

Sua utilidade baseia-se principalmente na possibilidade de ampliação da atuação de uma equipe multidisciplinar, composta por pedagogos, psicólogos e profissionais vinculados à educação e à educação especial, no processo de identificação e atendimento educacional especializado ao aluno com AH/S (Fleith, 2007). Isso porque, no processo de identificação, sem dúvida, os professores apresentam-se como profissionais que possuem maior proximidade e afinidade com os alunos, de modo a constituírem-se em fonte importante de consulta no processo de reconhecimento dos diferentes domínios da superdotação (Cohen, Ambrose, \& Powell, 2000; Miller \& Cohen, 2012), a qual não é considerada quando se faz uso exclusivo de testes psicológicos para avaliação. A nomeação por professores constitui uma alternativa às formas tradicionais de identificação das $\mathrm{AH} / \mathrm{S}$, usualmente baseadas em medidas psicométricas e de desempenho (Barbosa, Schelini \& Almeida, 2012).

No processo de desenvolvimento de uma medida psicológica, é importante que sejam conduzidos diferentes estudos a fim de verificar as evidências de validade do instrumento que está sendo construído. O estudo aqui apresentado busca complementar outros já desenvolvidos com o instrumental, a saber: evidências de validade baseadas no conteúdo (Nakano, Campos, \& Santos, 2016) e evidências de validade concorrente com base nas relações com variáveis externas (Nakano, Gozzoli, Alves, \& Campos, 2016). Isso porque, 
conforme apontado por Ambiel e Carvalho (2017), a busca por evidências de validade deve ser considerada dentro de um processo contínuo e ininterrupto, sendo que as primeiras evidências a serem alcançadas, após as evidências de conteúdo, devem ser as evidências de validade baseadas na estrutura interna.

Ainda segundo os autores, esse tipo de investigação visa verificar a relação entre os itens do instrumento a fim de representar a composição teórica do construto. Para isso, o pesquisador deve conduzir estudos que possibilitem a compreensão dos diferentes agrupamentos dos itens de modo que, a partir dessa observação dos dados, será possível inferir a existência de um traço latente, ou ainda, do construto investigado (Primi, 2011). No caso aqui específico, de $\mathrm{AH} / \mathrm{S}$.

Para esse tipo de evidência de validade, a análise fatorial exploratória (AFE) ou análise fatorial confirmatória (AFC) são as mais utilizadas pelos pesquisadores (Ambiel \& Carvalho, 2017) tendo sido escolhida, para o presente estudo, a AFC. Tal análise permite que o pesquisador realize o teste confirmatório da estrutura psicométrica do instrumento desenvolvido de acordo com o modelo teórico que a embasou (Pilati \& Laros, 2007).

Considerando-se que a Triagem Indicadores de Altas Habilidades/Superdotação (TIAH//S) encontra-se em processo de construção, mostra-se fundamental o desenvolvimento de estudos dessa natureza. O instrumento foi desenvolvido a partir de uma perspectiva teórica na qual se entende que as AH/S se constitui em um fenômeno multidimensional, composto por diferentes aspectos nos quais uma das seguintes habilidades acima da média pode se manifestar: capacidade intelectual geral, habilidades acadêmicas, liderança, criatividade e talento artístico, de forma isolada ou combinada (Brasil, 2010), embasado na Gifted Rating Scale - school form (GRS-S, Pfeiffer \& Jarosewich, 2003), avaliando as mesmas áreas que tal escala.

Conforme orientações apresentadas na literatura especializada (Lara \& Alexis, 2014; Muthén \& Muthén, 2012), foram avaliados os seguintes índices: qui-quadrado $\left(\chi^{2}\right)$, graus de liberdade $(g l)$, Comparative Fix Index (CFI), Tucker Lewis Index (TLI) e Root Mean Square Error of Approximation (RMSEA). No que se refere à interpretação dos dados, Pilati e Laros (2007) afirmam que é importante que os valores relacionados ao CFI e ao TLI estejam compreendidos entre 0,90 e 0,95 para que sejam entendidos como indicadores de ajuste suficiente. Enquanto, valores superiores a 0,95 devem ser considerados como indicadores de bons ajustes. Quanto aos valores do RMSEA, os autores supracitados afirmam que são esperados valores inferiores a 0,08 a fim de que o modelo seja considerado como adequado. Dessa forma, tendo em vista o objetivo de compreender a estrutura fatorial do instrumento, entende-se que a AFC oferece recursos mais apropriados para este estudo.

\section{Método}

\section{Participantes}

A amostra foi composta por 216 crianças e adolescentes com idades entre 8 e 15 anos $(M=11,97 ; D P=2,05)$, sendo 102 do sexo feminino (47,2\%), estudantes de escolas regulares de ensino, localizadas em cinco diferentes cidades, sendo duas no Estado de São Paulo $(n=82)$, uma no Sul $(n=89)$, uma no Distrito Federal $(n=3)$ e outra no Maranhão $(n=42)$.

\section{Instrumento}

Para a condução do presente estudo, foi utilizado o instrumento TIAH/S, composto por 42 afirmativas, divididas em cinco áreas do desenvolvimento. A área chamada Capacidade Intelectual Geral é composta por oito itens que avaliam as habilidades mentais verbais e não verbais dos estudantes, capacidades ou competências incluindo facilidades relacionadas à aprendizagem de ideias abstratas, resolução de problemas, raciocínio, velocidade mental e memória. A área Habilidades Acadêmicas Específicas contempla nove itens e compreende as habilidades em lidar com dados, fatos e materiais relacionados ao conteúdo escolar, como a leitura avançada e/ou proficiência para a leitura, matemática e outros aspectos do currículo escolar. A área Liderança tem oito itens que envolvem a capacidade de motivar pessoas em direção a uma meta comum, incluindo comportamentos de ouvir bem, agir com responsabilidade, manter-se calmo e sereno durante divergências, inspirar confiança e cooperação. A Criatividade mede, por meio de oito itens, a habilidade para pensar, agir ou produzir ideias inovadoras ou produtos únicos, originais ou novos, expressa pela maneira como o indivíduo resolve os problemas, experimenta novas ideias ou brinca imaginativamente. Por fim, a área de Talento Artístico, composta por nove itens, avalia o potencial ou evidência de capacidade superior nas áreas de atuação/teatro, dança, desenho, pintura, escultura, canto, música, dentre outras relacionadas à área artística, expresso pela forma como o indivíduo se aproxima de atividades lúdicas, completa tarefas e/ou trabalha com materiais de arte.

O instrumento é respondido pelo professor, sendo que a tarefa envolve assinalar a alternativa que mais bem descrever o aluno que está sendo avaliado, dentre as opções: abaixo da média se comparado a estudantes da mesma turma; nível da habilidade igual à de outros estudantes; acima da média apresentada pelos outros estudantes quando comparado a outros alunos da mesma idade. Caso o professor não se sinta apto para sinalizar o nível de comportamento que melhor descreve o aluno, ele poderá, na última coluna, marcar um X em "incapaz de julgar”.

\section{Procedimentos}

Os instrumentos foram respondidos pelos professores, sendo um para cada aluno avaliado. Um total de 
74 professores participaram do processo, respondendo entre duas e cinco avaliações, a partir da orientação que solicitava que eles indicassem alunos que possuíam alguma habilidade acima da média quando comparado com outros estudantes da mesma idade ou que se destacavam em alguma área e, em igual número, estudantes que não se destacavam na sala de aula. Os professores deveriam conhecer o aluno há, pelo menos, seis meses e ser professor regular da sala de aula (excluindo-se os substitutos).

Ora o processo de resposta foi feito durante a coleta de dados realizada com os alunos (para outra pesquisa de busca por evidências de validade do tipo convergente com uma bateria de avaliação de altas habilidades/superdotação, também em processo de construção), ora os professores responderam à escala em momento mais conveniente, devolvendo-os, posteriormente, em dia e horário combinado com a pesquisadora.

\section{Análise de dados}

No que diz respeito à análise dos dados, foram computadas as pontuações de cada um dos participantes e, em seguida, foi construído um banco de dados contendo as informações sociodemográficas (sexo, idade, nível de escolaridade e tipo de escola), bem como as respostas a cada um dos itens de todos os participantes. Inicialmente, com auxílio do programa RStudio 3.4 (R Core Team,
2018) utilizando-se o pacote Lavaan (Rosseel, 2014), empregou-se a análise fatorial confirmatória (AFC). Considerando as orientações referentes ao tipo de método de estimação, optou-se por utilizar o método denominado Diagonal Weighted Least Squares (DWLS). Foram verificadas as diferenças entre os índices de ajustamento: $\chi^{2}, g l$, CFI, TLI e RMSEA. Após a realização dessas ações, verificou-se a precisão do instrumento por meio da análise dos coeficientes de alfa de Cronbach. Para isso, utilizou-se o software Statistical Package for the Social Sciences (SPSS) versão 22.

\section{Resultados}

Estimou-se o valor do $\chi^{2}(809)=0,0001$, verificando-se que o CFI=0,991 e TLI=0,990 apresentaram bom ajuste, de acordo com os critérios adotados (Pilati \& Laros, 2007). Por sua vez, o RMSEA $=0,080$ apresentou valor igual ao valor mínimo sugerido 0,08 , de modo que é possível verificar a adequação do modelo. Por meio desses resultados, foi possível confirmar a estrutura fatorial da TIAH/S de acordo com o modelo proposto, composto por cinco fatores, cujas cargas fatoriais dos itens para os fatores teóricos são apresentadas na Tabela 1. Importante destacar que, na análise conduzida, o primeiro item de cada fator teve sua carga fatorial fixada em 1,000.

Tabela 1

Valores das Cargas Fatoriais para os Fatores Específicos

\begin{tabular}{|c|c|c|c|c|c|}
\hline Item & $\begin{array}{c}\text { Fator } 1 \\
\text { Capacidade } \\
\text { Intelectual }\end{array}$ & $\begin{array}{c}\text { Fator } 2 \\
\text { Habilidades } \\
\text { Acadêmicas }\end{array}$ & $\begin{array}{c}\text { Fator } 3 \\
\text { Liderança }\end{array}$ & $\begin{array}{c}\text { Fator } 4 \\
\text { Criatividade }\end{array}$ & $\begin{array}{c}\text { Fator } 5 \\
\text { Talento } \\
\text { Artístico }\end{array}$ \\
\hline 1 & 1,000 & & & & \\
\hline 2 & 0,866 & & & & \\
\hline 3 & 0,936 & & & & \\
\hline 4 & 0,889 & & & & \\
\hline 5 & 0,899 & & & & \\
\hline 6 & 0,869 & & & & \\
\hline 7 & 0,903 & & & & \\
\hline 8 & 0,886 & & & & \\
\hline 9 & & 1,000 & & & \\
\hline 10 & & 0,917 & & & \\
\hline 11 & & 0,889 & & & \\
\hline 12 & & 0,754 & & & \\
\hline 13 & & 0,814 & & & \\
\hline 14 & & 0,806 & & & \\
\hline 15 & & 0,891 & & & \\
\hline 16 & & 0,823 & & & \\
\hline 17 & & 0,846 & & & \\
\hline 18 & & & 1,000 & & \\
\hline 19 & & & 0,909 & & \\
\hline 20 & & & 0,834 & & \\
\hline 21 & & & 0,895 & & \\
\hline 22 & & & 0,849 & & \\
\hline
\end{tabular}


Tabela 1 (continuação) Valores das Cargas Fatoriais para os Fatores Específicos

\begin{tabular}{|c|c|c|c|c|c|}
\hline Item & $\begin{array}{c}\text { Fator } 1 \\
\text { Capacidade } \\
\text { Intelectual }\end{array}$ & $\begin{array}{c}\text { Fator } 2 \\
\text { Habilidades } \\
\text { Acadêmicas }\end{array}$ & $\begin{array}{c}\text { Fator } 3 \\
\text { Liderança }\end{array}$ & $\begin{array}{c}\text { Fator } 4 \\
\text { Criatividade }\end{array}$ & $\begin{array}{l}\text { Fator } 5 \\
\text { Talento } \\
\text { Artístico }\end{array}$ \\
\hline 23 & & & 0,893 & & \\
\hline 24 & & & 0,808 & & \\
\hline 25 & & & 0,893 & & \\
\hline 26 & & & & 1,000 & \\
\hline 27 & & & & 0,870 & \\
\hline 28 & & & & 0,819 & \\
\hline 29 & & & & 0,924 & \\
\hline 30 & & & & 0,919 & \\
\hline 31 & & & & 0,847 & \\
\hline 32 & & & & 0,816 & \\
\hline 33 & & & & 0,733 & \\
\hline 34 & & & & & 1,000 \\
\hline 35 & & & & & 0,936 \\
\hline 36 & & & & & 0,729 \\
\hline 37 & & & & & 0,837 \\
\hline 38 & & & & & 0,844 \\
\hline 39 & & & & & 0,900 \\
\hline 40 & & & & & 0,878 \\
\hline 41 & & & & & 0,717 \\
\hline 42 & & & & & 0,833 \\
\hline
\end{tabular}

Conforme pode ser visualizado, os valores das cargas fatoriais dos oito itens que compõem o Fator 1 - Capacidade Intelectual Geral, variaram entre 0,869 e 0,936 . Segundo o modelo teórico adotado, tal fator se refere à presença de habilidades e competências gerais, as quais envolvem a capacidade de processar informações e resolver problemas, estando relacionada à memória, capacidade verbal, raciocínio e pensamento abstrato.

O Fator 2, denominado Habilidades Acadêmicas Específicas, é composto por nove itens, cujas cargas fatoriais oscilaram entre 0,754 e 0,917 . Ele engloba itens cujo conteúdo envolve domínio e destaque em alguma área específica de conhecimento, tal como matemática, linguagem, leitura, escrita, dentre outras. Em relação ao Fator 3, Liderança, seus oito itens apresentaram carga fatorial que oscilou entre 0,808 e 0,909 e dizem respeito à capacidade de destacar-se dentre os colegas de classe, de liderar e tomar decisões e de influenciar os demais.

O Fator 4, intitulado Criatividade, é composto por oito itens, que apresentaram carga fatorial entre $0,733 \mathrm{e}$ 0,924, representando uma habilidade acima da média em relação à capacidade de criar formas originais e diferentes de lidar com os problemas e situações, presença de flexibilidade de pensamento, grande número de ideias, imaginação e curiosidade. Por fim, o Fator 5, referente ao Talento Artístico, ficou composto por nove itens com carga fatorial entre 0,7171 e 0,936 , os quais representam interesses e talentos em áreas como música, dança, arte, teatro, desenho, pintura.

A partir dos resultados da AFC, foi possível confirmar a multidimensionalidade da escala de Triagem de Indicadores de Altas habilidades/Superdotação, dentro das cinco áreas que ela se propõe a avaliar. Diante do fato de que nenhum dos itens analisados apresentou carga fatorial menor do que 0,30 , cujo valor justificaria sua exclusão, a versão final do instrumento ficou composta por 42 itens. Em seguida, a precisão do instrumento foi calculada por meio da consistência interna. Os valores dos coeficientes de alfa de Cronbach são apresentados na Tabela 2, a seguir.

A partir dos resultados, pode-se verificar que todos os fatores apresentaram precisão adequada, com valores oscilando entre 0,898 e 0,946 . Igualmente, a precisão geral da escala foi de 0,969 . 
Tabela 2

Valores do Alfa de Cronbach por fator da TIAH/S

\begin{tabular}{|c|c|c|}
\hline Fator & $\alpha$ & $\mathrm{N}^{\circ}$ de itens \\
\hline Capacidade Intelectual Geral & 0,946 & 8 \\
\hline Habilidades Acadêmicas Específicas & 0,898 & 9 \\
\hline Liderança & 0,929 & 8 \\
\hline Criatividade & 0,898 & 8 \\
\hline Talento Artístico & 0,912 & 9 \\
\hline Total & 0,969 & 42 \\
\hline
\end{tabular}

\section{Discussão}

Os resultados da AFC se mostraram favoráveis ao modelo teórico adotado como base para a construção da escala. A partir das análises conduzidas, foi possível verificar que a TIAH/S confirmou um modelo multidimensional das altas habilidades/superdotação, reconhecido em diferentes países (Feldman, 2000; Gagné, 2005). Do mesmo modo, confirmou o ajuste do instrumento para avaliação das cinco diferentes áreas propostas teoricamente, a saber: capacidade intelectual geral, habilidades acadêmicas específicas, criatividade, liderança e talento artístico. Pode-se dizer que os achados também vão ao encontro da compreensão do fenômeno que embasa as políticas públicas brasileiras (Brasil, 2010), bem como a avaliação das mesmas áreas da escala tomada como base para a construção da TIAH/S (Pfeiffer \& Jarosewich, 2003).

A confirmação da estrutura fatorial da TIAH/S enquanto uma medida multidimensional acompanha uma tendência apresentada em vários outros instrumentos internacionais, do mesmo tipo (escala) e com a mesma finalidade (identificação das AH/S). Dentre alguns exemplos, pode-se citar a The Gifted Evaluation Scale (McCarney \& Anderson, 1998) a qual avalia cinco áreas: habilidade intelectual, criatividade, habilidade acadêmi$\mathrm{ca}$, artes visuais e liderança, além de uma escala opcional de motivação. A Scales for Identifying Gifted Students (Ryser \& McConnell, 2004) incluem sete subescalas: habilidade intelectual geral, artes e linguagem, matemática, ciências, estudos sociais, criatividade e liderança. A Gifted and Talented Evaluation Scales (Gilliam, Carpenter, \& Christensen, 1996) também incluem subescalas de habilidade intelectual, habilidade acadêmica, criatividade, liderança e talento artístico. Por fim, a Gifted Rating Scales (Pfeiffer \& Jarosewich, 2003), tomada como base para a construção da TIAH/S também avalia seis escalas: habilidade intelectual, habilidade acadêmica, criatividade, talento artístico, liderança e motivação.

Em relação a este último instrumento, suas versões traduzidas e adaptadas para diferentes países também apresentaram a mesma estrutura fatorial. Na versão em espanhol (Rosado, Pfeiffer, \& Petscher, 2008) seis fatores foram encontrados por meio da análise fatorial confirmatória. A versão americana também indicou a adequação do modelo composto por seis escalas ( $\mathrm{Li}$, Lee, Pfeiffer, \& Petscher, 2008; Margulies \& Floyd, 2004; Pfeiffer \& Blei, 2008; Petscher \& Li, 2008). A invariância da escala em diferentes países (Estados Unidos, Porto Rico, China, Coreia do Sul e Turquia) também apontou para uma estrutura multidimensional, composta por cinco fatores em todos os países avaliados, de maneira a indicar que seus resultados podem ser interpretados de maneira equivalente nas culturas mencionadas ( $\mathrm{Li}$, Lee, Pfeiffer, Kamatam, \& Rosado, 2009).

A relevância dos resultados aqui relatados se ampara na importância de se apresentar uma ferramenta que consegue apontar para comportamentos e indicadores de AH/S em diferentes áreas, considerando-se, principalmente, o fato de que os superdotados não podem ser considerados um grupo homogêneo. Nesse sentido, a literatura tem apontado que, se quiser conhecer e entender as $\mathrm{AH} / \mathrm{S}$, é necessário focar nas diferenças intra individuais e interindividuais para poder caracterizar, de forma adequada, o indivíduo nessa condição (Costa \& Lubart, 2016).

Somado aos resultados obtidos anteriormente por outros estudos já conduzidos com o instrumental, pode-se afirmar que os dados aqui apresentados acrescentam evidências de validade à escala, de modo a apontar sua capacidade de englobar distintos construtos psicológicos que estão envolvidos na compreensão do fenômeno (Gallagher, 2008). O estudo aqui relatado adquire importância ao contribuir para a investigação de uma fonte adicional de evidências de validade, bem como da precisão, de um instrumento desenvolvido especificamente para a avaliação dessa população minoritária, com o objetivo de sanar, em partes, as lacunas em relação à ausência de instrumentos com esse propósito (Barbosa et al., 2012).

\section{Considerações Finais}

Almeja-se que, futuramente, a condução de outros estudos voltados à investigação das qualidades psicométricas da TIAH/S possa também apontar para resultados positivos, de modo que, futuramente, o instrumental possa ser disponibilizado para uso profissional. Assim, a ferramenta poderá ser utilizada com o propósito de melhoria 
do processo de encaminhamento dos estudantes para a avaliação psicológica e posterior confirmação do quadro de altas habilidades/superdotação. Consequentemente, espera-se que seus resultados possam auxiliar no planejamento do atendimento educacional especializado, desenvolvimento de forças e potenciais, bem como a prevenção de possíveis dificuldades emocionais e sociais que a ausência de identificação pode provocar. Tais medidas podem ser consideradas parte das premissas que embasam o movimento da Psicologia Positiva, de busca pela promoção de um desenvolvimento sadio, por meio do fortalecimento dos recursos pessoais.

\section{Referências}

Almeida, L. S., Araújo, A. M., Sainz-Gómez, M., \& Prieto, M. D. (2016). Challenges in the identification of giftedness: Issues related to psychological assessment. Anales de Psicologia, 32(3), 621-627. Recuperado de http://hdl.handle.net/11328/1758

Almeida, L. S., Fleith, D. S., \& Oliveira, E. P. (2013). Sobredotação: Respostas educativas. Braga: ADIPSICEDUC.

Ambiel, R. A. M., \& Carvalho. L. F. (2017). Validade e precisão de instrumentos de avaliação Psicológica. Em M. R. C. Lins \& J. C. Borsa (Eds.), Avaliação Psicológica: aspectos teóricos e práticos (pp. 115-125). Petrópolis, RJ: Vozes.

Barbosa, A. J. G., Schelini, P. W., \& Almeida, L. C. (2012). Medidas de dotação e talento: Produção científica em Psicologia (2006-2011). Em E. Boruchovitch, A. A. A. Santos \& E. Nascimento (Eds.), Avaliação psicológica nos contextos educativo e psicossocial (pp. 33- 52). São Paulo: Casa do Psicólogo.

Brasil (2010). Políticas públicas para alta habilidade/superdotação. Recuperado de www.senado.gov.br/web/comissoes/CE/AP/AP20080626 superdotados $\mathrm{Cl} \% \mathrm{C} 3 \% \mathrm{~A} 1$ udiaGriboski.pdf

Chagas-Ferreira, J. F. (2014). As características socioemocionais do indivíduo talentoso e a importância do desenvolvimento de habilidades sociais. Em A. M. R. Virgolim (Eds.), Altas habilidades/superdotação, inteligência e criatividade: uma visão multidisciplinar (pp. 283-308). Campinas: Papirus.

Chagas-Ferreira, J. F., \& Sousa, R. A. R. (2018). O desenvolvimento socioemocional de superdotados: Descrevendo singularidades e identificando possibilidades de atendimento. Em F. H. R. Piske, T. Stoltz, C. Costa-Lobo, A. Rocha, \& Vásquez-Justo, E. (Eds.), Educação de superdotados e talentosos: emoção e criatividade (pp. 127-138). Curitiba: Juruá.

Cleveland, L. M. (2017). Examining the relationship between Gifted Behavior Rating Scores and student academic performance. Dissertation. Concordia University, Portland. Recuperado de https://commons.cu-portland.edu/cgi/viewcontent.cgi?referer=https:/www.google.com/\&https redir $=1 \&$ article $=1030 \&$ context $=$ edudissertations

Cohen, L. M., Ambrose, D., \& Powell, W. N. (2000). Conceptual foundations and theoretical lenses for the diversity of giftedness and talent. Em K. A. Heller, F. J. Mönks, R. Subotnik, \& R. J. Sternberg (Eds.), International handbook of research and development of giftedness and talent (pp.331-344). Oxford, England: Pergamon.

Costa, M. P. \& Lubart, T. I. (2016). Gifted and talented children: Heterogeneity and individual differences. Anales de Psicologia, 32(3), 662671. doi: 10.6018/analesps.32.3.259421

Feldman, D. H. (2000). The development of creativity. Em R.J. Sternberg (Ed.), Handbook of creativity (pp. 169-189). Cambridge: Cambridge University Press.

Fleith, D. S. (2007). Altas habilidades e desenvolvimento socioemocional. Em D. S. Fleith \& E. M. L. S. Alencar (Eds.), Desenvolvimento de talentos e altas habilidades: orientação a pais e professores (pp.41-50). Porto Alegre: Artmed.

Freitas, M. F. R. L., Schelini, P. W., \& Pérez, R. (2017). Escala de identificação de dotação e talento: Estrutura e consistência internas. Psico-USF, 22(3), 473-484. doi: 10.1590/1413-82712017220308

Gagné, F. (2005). From gifted to talents: the DMGT as a developmental model. Em R. J. Sternberg \& J. E. Davidson (Eds.), Conceptions of Giftdeness. 2 ed. (pp. 98-120). New York: Cambridge University Press.

Gallagher, J. J. (2008). Psychology, psychologist, and gifted students. Em S. Pfeiffer (Ed.). Handbook of giftedness in children: Psycho-educational theory, research and best practices (pp. 1-11). New York: Springer.

Gilliam, J. E. Carpenter, B. O., \& Christensen, J. R. (1996). Gifted and Talented Evaluation Scales. Austin, TX: PRO-ED.

Gray, R. G. (2007). Reliability and validity of the UNIT - Gifted Screening Scale (Tese de doutorado). University of Tennessee. Recuperado de https://trace.tennessee.edu/cgi/viewcontent.cgi? referer=https:/www.google.com/\&httpsredir=1\&article=5923\&context=utk_graddiss

Guenther, Z. (2006). Capacidade e talento: Um programa para a escola. São Paulo: EPU.

Guimarães, T. G. (2007). Avaliação Psicológica de alunos com altas habilidades. Em D. S. Fleith \& E. M. L. S. Alencar (Eds.), Desenvolvimento de talentos e alta habilidades - orientação a pais e professores (pp. 79-86). Porto Alegre: Artmed.

Heller, K. A. (2004). Identification of gifted and talented students. Psychology Science, 46(3), 302-323. Recuperado de https://psycnet.apa.org/ record/2005-05211-002

Irueste, P., Saco, A., \& Nicolás, F. (2018). Dificultades socioemocionais reportadas por los padres y madres de niños y niñas dotados y talentosos, consultantes del Servicio de Neuropsicologia, Área Infantil, em Córdoba, Argentina. Em F. H. R. Piske, T. Stoltz, C. Costa-Lobo, A. Rocha \& Vásquez-Justo, E. (Eds.), Educação de superdotados e talentosos: emoção e criatividade (pp. 75-88). Curitiba: Juruá.

Kaufman, J. C., Plucker, J. A., \& Russell, C. M. (2012). Identifying and assessing creativity as a component of giftedness. Journal of Psychoeducational Assessment, 30(1), 60-73. doi: 10.1177/0734282911428196

Kerr, B., \& Sodano, S. (2003) Career assessment with intellectually gifted students. Journal of Career Assessment, 11, 168-186. doi: $10.1177 / 1069072703011002004$

Lara, D. \& Alexis, S. (2014). ¿Matrices policóricas/tetracóricas o matrices Pearson? Un estudio metodológico. Revista Argentina de Ciencias del Comportamiento, 6(1), 39-48. doi: 10.30882/1852.4206.v6.n1.6357

Li, H., Lee, D., Pfeiffer, S., Kamata, A., \& Rosado, J. (2009). measurement invariance for the gifted rating scales-school form across five cultural groups. School Psychology Quaterly, 24(3), 186-198. doi:10.1037/a0017382 
Li, H., Lee, D., Pfeiffer, S. I., \& Petscher, Y. (2008). Parent ratings using the Chinese version of the Parent Gifted Rating Scales School form reliability and validity for Chinese students. Educational and Psychological Measurement, 68, 659-675. doi: 10.1177/0013164407313365.

Margulies, A. S., \& Floyd, R. G. (2004). Book review: Gifted Rating Scales (GRS). Journal of Psychoeducational Assessment, 22, 275-282. doi: $10.1177 / 073428290402200306$

Martins, B. A., Pedro, K. M., Ogeda, C. M. M., Silva, R. C., Koga, F. O., \& Chacon, M. C. M. (2016). Altas habilidades/superdotação: Estudos no Brasil. Journal of Research in Special Educational Needs, 16(1), 135-139. doi: 10.1111/1471-3802.12275

McCarney, S. B., \& Anderson, P. D. (1998). The Gifted Evaluation Scale (2nd ed.): Technical manual. Columbia, MO: Hawthorne Educational Services.

Miller, E. M., \& Cohen, L. M. (2012). Engendering talent in others: Expanding domains of giftedness and creativity. Roeper Review, 34, 104113. doi: $10.1080 / 02783193.2012 .660684$

Muthén, L. K., \& Muthén, B. O. (2012). Mplus User's Guide. Seventh Edition. Los Angeles, CA: Muthén \& Muthén.

Nakano, T. C., Campos, C. R., \& Santos, M. V. (2016). Escala de avaliação das altas habilidades/superdotação - versão professor: Validade de conteúdo. Estudos Interdisciplinares em Psicologia, 7(1), 103-123. Recuperado de http://pepsic.bvsalud.org/scielo.php?pid=S2236$64072016000100007 \&$ script $=$ sci_abstract\&tlng $=$ es

Nakano, T. C., Gozzoli, M. Z., Alves, R. J. R., \& Campos, C. R. (2016). Investigación de la eficácia de uma escala de evaluación de altas habilidades - versión profesor. Revista de Estudios y Experiencias em Educacion, 15(29), 83-94. doi: 10.21703/rexe.20162983945

Negrini, T., \& Freitas, S. N. (2008). A identificação e a inclusão de alunos com características de altas habilidades/superdotação: Discussões pertinentes. Revista Educação Especial, 32, 273-284. Recuperado de https://periodicos.ufsm.br/educacaoespecial/article/view/103

Petscher, Y., \& Li, H. (2008). Measurement invariance of the Chinese gifted rating scales: Teacher and parent forms. Journal of Psychoeducational Assessment, 26, 274-286. doi: 10.1177/0734282907303873

Pfeiffer, S. I. (2018). Understanding success and psychological well-being of gifted kids and adolescents: Focusing on strengths of the heart. Estudos de Psicologia (Campinas), 35(3), 259-263. doi: 10.1590/1982-02752018000300004

Pfeiffer, S., \& Blei, S. (2008). Gifted identification beyond the IQ test: Rating scales and other assessment procedures. Em S. Pfeiffer (Eds.), Handbook of giftedness in children: Psycho- educational theory, research and best practices (pp. 177-198). New York: Springer.

Pfeiffer, S., \& Jarosewich, T. (2003). Gifted Rating Scales - Manual. San Antonio/TX: Pearson.

Pilati, R., \& Laros, J. A. (2007). Modelos de equações estruturais em psicologia: Conceitos e aplicações. Psicologia: Teoria e Pesquisa, 23(2), 205 - 216. doi: 10.1590/S0102-37722007000200011

Piske, F. H. R. (2017). Avanços cognitivos e socioafetivos de uma criança superdotada: Um estudo de caso. Em F. H. R. Piske, C. L. B. Vestena, T. Stoltz, J. M. Machado, A. A. O. M. Barby, S. Bahia \& S. P. Freitas (Eds.), Processos afetivos e cognitivos de superdotados e talentosos (pp.137-164). Curitiba: Editora Prismas.

Pocinho, M. (2009). Superdotação: Conceitos e modelos de diagnóstico e intervenção psicoeducativa. Revista Brasileira de Educação Especial, 15(1), 3-14. doi: 10.1590/S1413-65382009000100002

Prado, R. M., \& Fleith, D. S. (2017). O papel do aconselhamento psicológico no desenvolvimento afetivo do aluno superdotado. Em F.H.R. Piske et al. (Eds.), Processos afetivos e cognitivos de superdotados e talentosos (pp.209-224). Curitiba: Prisma.

Primi, R. (2011). Responsabilidade ética no uso de padrões de qualidade profissional na avaliação psicológica. Em Conselho Federal de Psicologia (Eds.), Ano da Avaliação Psicológica - Textos Geradores (pp. 53 - 58). Brasília, DF: Conselho Federal de Psicologia.

R Development Core Team (2014) R: A Language and Environment for Statistical Computing. R Foundation for Statistical Computing, Vienna, Austria. R Foundation for Statistical Computing, Vienna.

Renzulli, J. S. (2004) O que é esta coisa chamada superdotação e como a desenvolvemos? Retrospectiva de vinte e cinco anos. Revista Educação, 27(1), 75-134. Recuperado de https://www.marilia.unesp.br/Home/Extensao/papah/o-que-e-esta-coisa-chamada-superdotacao.pdf

Renzulli, J. S., Siegle, D., Reis, S. M., Gavin, M. K., \& Reed, R. E. S. (2009). An investigation of the reliability and factor structure of four new scales for rating the behavioral characteristics of superior students. Journal of Advanced Academics, 21(1), 84-108. Recuperado de https://files.eric.ed.gov/fulltext/EJ880576.pdf

Rosado, J., Pfeiffer, S., \& Petscher, Y. (2008). The reliability and validity of an Spanish translated version of the Gifted Rating Scales. Gifted and Talented International, 23(1), 105-114. Recuperado de https://www.ncbi.nlm.nih.gov/pmc/articles/PMC4574492/

Rosseel, Y. (2014). The lavaan tutorial. Department of Data Analysis: Ghent University.

Ryser, G. R., \& McConnell, K. (2004). Scales for Identifying Gifted Students: Ages 5 through 18. Waco, TX: Prufrock Press.

Sabatella, M. L. P. (2008). Talento e superdotação: Problema ou solução? Curitiba: Ibpex.

Sahin, F. (2013). The scale for rating the behavioral characteristics of gifted and talented students: Study of factor structure, reliability and validity. Journal of Educational Sciences, 38, 119-132. doi: 10.15285/EBD.2013385570

Scorsolini-Comin, F., \& Santos, M. A. (2010). The scientific study of happiness and health promotion: An integrative literature review. Revista Latino-Americana de Enfermagem, 18(3), 472-479. doi: 10.1590/ S0104-11692010000300025

Valadez, M. D. (2010). Estados emocionales em niños y niñas con alta dotación. Em K. Morales Chacon (Ed.), Alta dotación y talentos en la niñez: aspectos básicos (pp.37-52). San José: C.R. Litografia e Imprenta.

Virgolim, A. M. (1997). O indivíduo superdotado: História, concepção e identificação. Psicologia: Teoria e Pesquisa, 13(1), $173-83$.

\section{Sobre os autores}

Tatiana de Cassia Nakano é psicóloga, doutora em Psicologia pela Pontifícia Universidade Católica de Campinas e, atualmente, é docente no Programa de Pós-Graduação Stricto Sensu em Psicologia da PUC-Campinas.

Karina da Silva Oliveira é psicóloga, doutora em Psicologia pela Pontifícia Universidade Católica de Campinas e, atualmente, cursa o pós-doutorado em Psicologia na Universidade São Francisco. 